\title{
LA INFLUENCIA DE GYÖRGY KEPES EN LA OBRA TEMPRANA DE JUAN NAVARRO BALDEWEG REALIZADA EN EL CENTER FOR ADVANCED VISUAL STUDIES DEL MIT (1971-1975)
}

\author{
Covadonga Lorenzo Cueva
}

En la obra temprana de Juan Navarro Baldeweg realizada durante su estancia en el Center for Advanced Visual Studies (CAVS) de/ Massachusetts Institute of Technology (MIT) bajo la tutela de György Kepes, se encuentran implícitos los principios que han guiado su modo de trabajar a lo largo de su vida. Él mismo ha reconocido la gran influencia que tuvieron sus años de estancia en el CAVS, haciendo referencia explícita a algunos de los artistas con los que allí trabajó y especialmente mencionando la influencia que ejerció Kepes sobre su forma de ver el mundo como un 'paisaje coherente' y sobre su modo de acercarse al entendimiento del medio físico. El artículo presenta los esquemas, proyectos, piezas, instalaciones e interiores que allí realizó, vinculándolos al contexto en el que se gestaron y mostrando de qué modo parte de su significación plástica respondió a su participación de este entorno colectivo.

Palabras clave: Navarro Baldeweg, György Kepes, arquitectura, entorno, arte Keywords: Navarro Baldeweg, György Kepes, Architecture, Environment, Art

Juan Navarro Baldeweg ha reconocido en varias ocasiones el interés que le suscitaron los escritos de György Kepes al comienzo de su trayectoria profesional, lo que le llevaría a solicitar en el año 1971 una estancia de investigación en el Center for Advanced Visual Studies (CAVS) del Massachusetts Institute of Technology (MIT), que se encontraba por entonces bajo la dirección de Kepes. En la lectura de algunas de sus obras más relevantes, como Language of Vision (1944) ${ }^{1}$, The New Landscape in Art \& Science $(1956)^{2}$ o la serie Vision and Value (1965-1966) ${ }^{3}$ es posible encontrar algunos de los fundamentos teóricos que se encontraba explorando en las actividades y proyectos que desarrollaba como director del CAVS. Para Kepes no existía una división clara entre el conocimiento científico y la visión poética o artística, sino que ambas convivían; ciencia y arte se complementaban y en su unidad, creía encontrar una solución a los problemas medioambientales que estaban afectando a las grandes ciudades en aquel momento. Con la creación del CAVS en 1967, Kepes se había propuesto crear un espacio en el que las aspiraciones creativas de una serie de artistas asociados al centro pudieran encontrar el apoyo de disciplinas científicas y tecnológicas, con el fin de desarrollar nuevas herramientas de intervención urbana, a partir de proyectos conjuntos de gran escala en los que interviniesen profesionales asociados a diferentes disciplinas. Al facilitarles un entorno de estas características confiaba en que los artistas podrían aprender de los modelos conceptuales que empleaban los científicos y las nuevas tecnologías que utilizaban los ingenieros, aportando por su parte, la inmediatez y la frescura propia de la visión poética que los artistas podían ofrecer del medio físico ${ }^{4}$.

La creación del CAVS supuso para Kepes la culminación de una larga trayectoria profesional que había iniciado en Hungría, su país natal, donde desde muy temprano entró en relación con los movimientos pictóricos de vanguardia. Durante algún tiempo abandonaría la pintura para dedicarse a explorar nuevos medios como la fotografía, el fotomontaje o el cine, trabajando con László Moholy Nagy. Ya en el MIT volvería a la pintura, disciplina que no abandonaría hasta el final de su vida y que desarrollaría desde entonces, en paralelo con su trabajo en las artes visuales donde continuó explorando nuevas tecnologías y medios de expresión plástica como la microfotografía, la espectroscopia, la holografía o el video. Durante sus años como director del CAVS, se centraría en propuestas plásticas que proponían la desmaterialización de la obra a nivel de entorno, así como la aplicación en el ámbito artístico de la ciencia, la tecnología y la electrónica, buscando trasladar las obras a una escala urbana. Con ello convertiría el centro en un lugar de experimentación de lo que se llegaría a denominar Environmental Art, un nuevo concepto que trascendería la noción de arte tradicionalmente referida a un objeto, para extenderla a un ámbito que ampliaba la propuesta plástica y al mismo tiempo, la circunscribía a un espacio provisto de su propia significación.
Todas las figuras, salvo aquellas en las que se especifique otra fuente, pertenecen al Center for Advanced Visual Studies Special Collection (CAVSSC), Massachusetts Institute of Technology, Program in Art, Culture \& Technology, Cambridge, Massachusetts.

1. KEPES, György, Language of Vision, Paul Theobald, Chicago, 1944

2. KEPES, G., The New Landscape in Art \& Science, Paul Theobald, Chicago, 1956

3. KEPES, G., Vision and Value Series, George Braziller, New York, 1965-1966.

4. Véase: KEPES, G., "Art talk on Imaginary Side", Evening Independent, St. Petesburg, Florida, 1974. 


\section{CONVIVENCIA ENTRE DISCIPLINAS ARTÍSTICAS}

5. "En mi inmersión en el medio cultural de Santander está la génesis de mi mundo imaginario y el amor por el ejercicio de distintas actividades expresivas, sin distinción entre las diversas artes". ZUAZNABAR, Guillermo (2011), "Una trayectoria", Juan Navarro Baldeweg. Conversaciones con estudiantes, Gustavo Gili, Barcelona.

6. En la academia de José Cataluña coincidiría con Eduardo Sanz, Agustín de Celis y Enrique Gran. En este ambiente conocería también al arquitecto Fernando Cuerno, al escultor Ramón Muriendas y al escritor Álvaro Pombo, quien recordaría esta época en un poema que le dedicaría años más tarde y que incluiría en su primer libro, Protocolos (1973).

7. A través de su padre, médico de profesión pero gran aficionado a la pintura, conoció al escultor José Villalobos, quien le pondría en contacto con el pintor, escultor y ceramista Miguel Vázquez Pesquera, en cuyo estudio se formaría también durante algunos años

8. En Autobiografía Intelectual (2012), Navarro Baldeweg reconoce que entre los libros que supusieron un gran estímulo para la afirmación de su vocación artística se encontraba $E l$ Estilo del siglo XX (1952), un regalo que recibió de su padre en 1954 así como los volúmenes sobre la Semana del Arte en Santillana del Mar publicados por la Escuela de Altamira, una corriente que ya entonces daba por sentada la autonomía del hecho artístico, el fin de la dependencia del arte con respecto a la realidad visual y su afirmación como lenguaje válido en sí mismo.

9. SOTA, Alejandro de la, "Prólogo", Grabados y Pinturas de Navarro Baldeweg, Librería-Galería Fernando Fe, Madrid, 1960.

10. ALEIXANDRE, Vicente, Antigua casa madrileña, Bedia, Santander, 1961

11. Para un análisis de la pintura de Navarro Baldeweg durante esta primera etapa consultar: MORENO, Ignacio, La Habitación Vacante de Juan Navarro Baldeweg. Análisis, origen e influencia de las ideas, mitos y conceptos de su experiencia artística aplicados a su arquitectura (Tesis Doctoral), Escuela Técnica Superior de Arquitectura, Universidad Politécnica de Madrid, 2004.

12. "Nuestra intención no es exponer las obras de los artistas como entidades aisladas sino crear, por emplear una cita, un 'sistema sinérgico' de todas las obras de arte incluidas, sin sacrificar sus cualidades individuales y su identidad. E énfasis estará en mostrar su interdependencia; una comunidad ambiental". Carta de Kepes a Smithson (30 de mayo de 1969), Robert Smithson Papers, Archives of American Art.

13. "La instalación (...) exhibe tres maneras típicas de significación en arquitectura: el espacio como teatro, como escultura y como geometría y la asimilación en un todo unitario y continuo de estos diferentes órdenes de simbolización". NAVA RRO BALDEWEG, Juan, "Piezas, instalaciones e interiores", EI medioambiente como espacio de significación (Memoria de investigación), Fundación Juan March, Madrid, 1975.

14. Bajo el título La habitación Vacante se agrupan tres exposiciones celebradas en 1976 que incluían dibujos, fotografía piezas e instalaciones: Luz y metales, celebrada en la Sala Vinçon de Barcelona; Útiles próximos, en la Galería Buades de Madrid y Habitaciones, en el Colegio Oficial de Arquitectos de Cataluña y Baleares.
A su llegada al CAVS en 1971, Navarro Baldeweg abandonaría también temporalmente la pintura, disciplina que durante los primeros años de su trayectoria profesional había empleado como instrumento analítico de investigación sobre el ámbito de la percepción, En el MIT, ampliaría esta búsqueda a través de una serie de esquemas, proyectos, piezas, instalaciones e interiores que le permitirían analizar las posibilidades de aplicación de éstas investigaciones en el ámbito de lo urbano. Si bien es cierto que su vinculación al CAVS fue determinante en su adscripción a nuevos medios de expresión plástica alejados de los que se podrían considerar convencionales, como la pintura, el dibujo, la escultura o el grabado, este interés por la experimentación con distintas disciplinas era ya una constante en su trabajo, cuyo origen él mismo ha situado en la libertad que vivió durante su formación en la infancia ligado al contexto artístico de Santander donde naciós. A los doce años comenzó sus primeros estudios de dibujo y pintura en la academia de José Cataluña ${ }^{6}$ y en los talleres de Miguel Vázquez ${ }^{7}$. De aquellos años recuerda la gran influencia de algunos libros con los que afirma haber asimilado la noción de estilo ${ }^{8}$, entendido como una cierta coherencia visual entre obras de diferentes disciplinas artísticas así como otras obras que le permitieron indagar sobre la dualidad del signo buscando un camino intermedio entre la figuración y la abstracción. Tras su paso por la Escuela de Bellas Artes de San Fernando en Madrid realizará su primera muestra individual en la Galería Fernando Fe, en marzo de 1960, con un catálogo con prólogo de Alejandro de la Sota9, a quien conoció al comienzo de sus estudios de Arquitectura que compaginaría desde entonces con la pintura. Tras una portada que realizó para ilustrar un poema de Aleixandre ${ }^{10}$ influido por el action painting iniciaría un camino hacia la abstracción pospictórica, con unas primeras obras en las que el gesto dejaba de tener importancia como expresión de lo corporal y en las que emplearía trazos y vertidos combinados con collages, influidas por el hard edge de Frank Stella y los combine paintings de Robert Rauschenberg ${ }^{11}$. Destacar también de aquel período las pinturas de vertidos inspiradas en los trabajos de Morris Louis y los collages, anunciando ya entonces el desplazamiento que se produciría en su obra hacia la consideración del entorno como medio de expresión plástica.

Una mirada sobre la temprana producción artística de Navarro Baldeweg previa a su estancia en el CAVS revela su interés por atender a la realidad empleando diferentes medios para tratar de responder al entorno con la misma variedad con la que se le presenta. Este interés es palpable también en las primeras propuestas que realizó a su llegada a los Estados Unidos, orientadas hacia la creación de sistemas artificiales, tras las cuales reorientaría su trabajo hacia una serie de interiores pensados para la activación del medio físico realizados a partir de mínimas actuaciones y cuya misión era alterar el espacio ontológicamente para transformarlo en un contenido perceptual que destacase particularidades insospechadas del mismo. Sus primeras instalaciones se presentarían en varias exposiciones colectivas, en las que se fomentaba el diálogo entre las obras de los distintos artistas según los presupuestos teóricos de Kepes, quien confiaba en la necesidad de hacer visibles dichas conexiones ${ }^{12}$. Posteriormente en sus interiores, Navarro Baldeweg presentaría también sus obras en convivencia, buscando la interacción con el espectador y su participación así como el establecimiento de conexiones entre todas ellas. Así, en su primera instalación, Interior I (1973), se aprecia la idea de integración en un mismo ámbito de varios medios expresivos: arquitectura, piezas y dibujos ${ }^{13}$. Desde entonces, sus sucesivos interiores agruparían distintas piezas elaboradas con diferentes soportes y medios, así como fotografías y dibujos. Una selección de obras pertenecientes a todos ellos, junto con las fotografías, los dibujos y los planos de su primer proyecto arquitectónico realizado a su regreso a Madrid, acabarían conviviendo también en la exposición retrospectiva $L u z$ $y$ metales $(1976)^{14}$.

\section{PERCEPCIÓN Y LENGUAJE VISUAL}

György Kepes creía firmemente en el papel social del arte y confiaba en que la búsqueda de soluciones a los problemas urbanos debía pasar por la realización de proyectos de gran escala orientados a la transformación de las ciudades. Este planteamiento tuvo su origen en los estudios que realizó con Kevin Lynch para la preparación del libro 

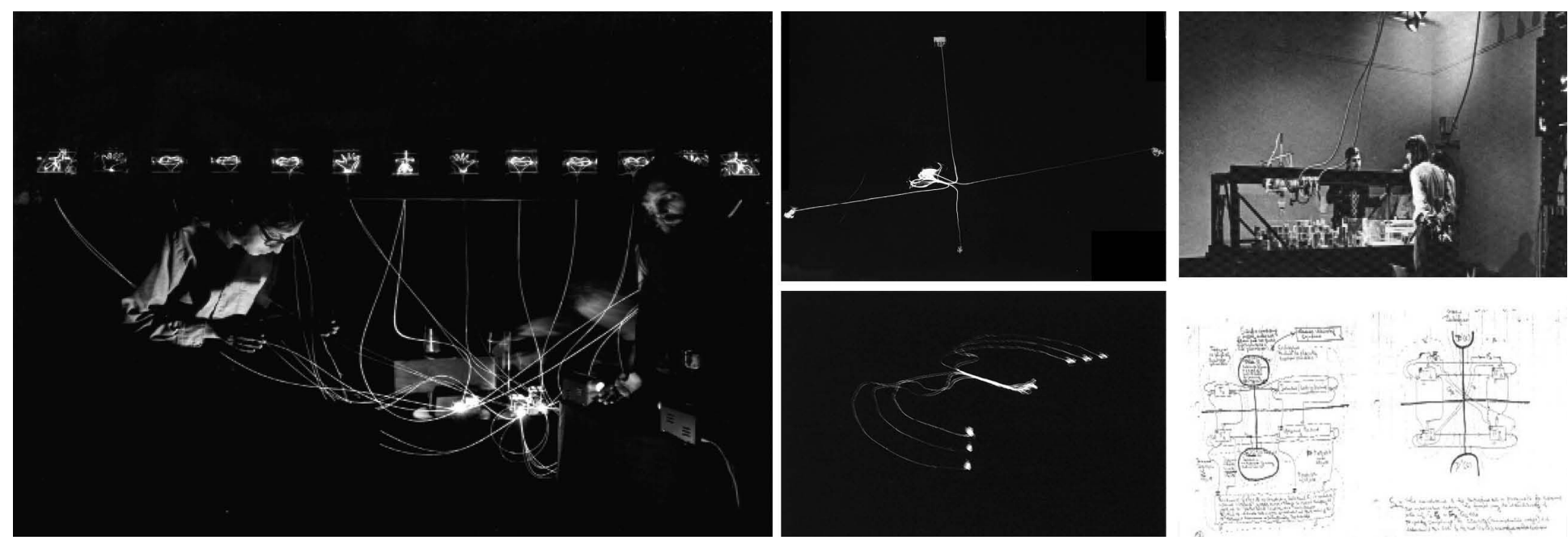

The Image of the City $(1960)^{15}$, que le llevarían más adelante a investigar sobre los fenómenos de la percepción, entendiéndolos como procesos de organización que implicaban la participación del espectador y en los que encontraba el fundamento esencial de todo proceso creativo ${ }^{16}$. En el CAVS, Kepes plantearía la posibilidad de elaborar un nuevo lenguaje de la visión que permitiese incorporar en la creación artística valores propios de la ciencia y la tecnología, para lo cual se propondría coordinar los proyectos de físicos, psicólogos, biólogos, matemáticos, ingenieros, filósofos, científicos e historiadores, así como pintores, escultores, artistas y diseñadores orientándolos hacia el estudio de las imágenes y su percepción desde las distintas disciplinas, así como al análisis de variables como la luz y la simetría y su relación con los procesos creativos y perceptivos, entendiendo ambos como procesos cognoscitivos que se producen en el individuo a partir del procesamiento cerebral que permite la selección, organización e interpretación de los estímulos procedentes del medio.

En esta misma tarea se encontraba entonces Navarro Baldeweg, quien ya en el artículo "El autómata residencial" ${ }^{17}$ escrito durante su estancia en el Centro de Cálculo de la Universidad de Madrid ${ }^{18}$, argumentaba la necesidad de adquirir un conocimiento más profundo de las operaciones mentales que se emplean en los procesos de diseño y analizaba los procesos que permiten construir las percepciones a partir de la experiencia, las expectativas y la motivación, defendiendo la capacidad de los procesos creativos para ampliar el umbral de percepción del individuo. Más adelante, en su primer proyecto en el CAVS, Gates from the Body (1971), hacía explícita esta preocupación por definir un nuevo lenguaje a partir de la destrucción de símbolos convencionales y la creación de otros nuevos que hicieran visibles los deseos e intereses de los ciudadanos ${ }^{19}$. En él, indagaría sobre la noción de ciudad como espacio significante y de comunicación y basándose en las teorías de Charles K. Ogden y Ivor A. Richards ${ }^{20}$ sobre la conformación del lenguaje por la interrelación entre significado, significante y referente, profundizaría en la asignación de significados conceptuales a los significantes del medio físico.

Con esta propuesta trataría de hacer visible una serie de gestos humanos mediante la disposición de una superficie a modo de lienzo en la que se visualizaban los símbolos asociados a dichas acciones (Fig. 1). En ella se hacían perceptibles los gestos de los participantes, que con cada acción activaban un sensor específico que transmitía esta información mediante fibra óptica hasta unas cajas con texturas que al iluminarse encendían el signo asociado al gesto. A partir de este primer proyecto, su trabajo se centraría en lo que suponía el acto de simbolizar, es decir, la asignación de significados a los significantes del medio físico, lo que identificaría con la apertura de ciertas 'puertas' físicas que conectarían la realidad física y la realidad conceptual. Se avanzaba aquí la existencia de dos ámbitos - uno real y otro imaginario- que desarrollaría unos años más tarde y denominaría 'habitaciones'. Ya en esta propuesta se genera un ámbito entendido como un umbral o una suerte de 'puertas' que permitían el paso de lo físico a lo inmaterial y latente bajo la morfología urbana.
Fig. 1. Juan Navarro Baldeweg, instalación Gates from the Body (1971), fotografías de Nishan Bichajian (izquierda y centro). Nicholas Negroponte, Seek (1970) (derecha). Fuente: Negroponte, Nicholas. 1973. The Architectural Machine: Toward a more Human Environment. Cambridge, Massachusetts, MIT Press.

15. LYNCH, Kevin, The Image of the City, MIT Press, Cambridge, Massachusetts, 1960.

16. KEPES, G., Language of Vision, op. cit., 1944.

17. NAVARRO BALDEWEG, J., SA1. Seminarios de Análisis y Generación Automática de Formas Arquitectónicas, Cuaderno 2, Centro de Cálculo de la Universidad de Madrid, 1972.

18. Una vez finalizada la tesis doctoral, Navarro Baldeweg recibió una beca del fondo IBM que le permitiría desarrollar entre 1969 y 1971 la línea de investigación iniciada en su tesis doctoral en las instalaciones del Centro de Cálculo de la Universidad de Madrid (actualmente Universidad Complutense de Madrid), donde se incorporó como investigador asociado y dirigió el programa sobre Composición Automática de Espacios Arquitectónicos (CAEA) que se inscribía dentro de los Seminarios de Análisis y Generación Automática de Formas Arquitectónicas coordinados por Javier Seguí de la Riva.

19. NAVARRO BALDEWEG, J., "Gates from the Body", Individual Works by Fellows at the Center for Advanced Visual Studies 1973-74, Center for Advanced Visual Studies Special Collection (CAVSSC), Massachusetts Institute of Technology, Program in Art, Culture \& Technology, Cambridge, Massachusetts.

20. Navarro Baldeweg cita explícitamente en la memoria del proyecto la siguiente obra: OGDEN, Charles K., RICHARDS, Ivor A., The Meaning of Meaning: a Study of the influence of Language upon Thought and on the Science of Symbolism, University of Cambridge, Reino Unido, 1923. Véase: NAVARRO BALDEWEG, J., El medio ambiente como espacio de significación, Madrid: Fundación Juan March, 1975. 

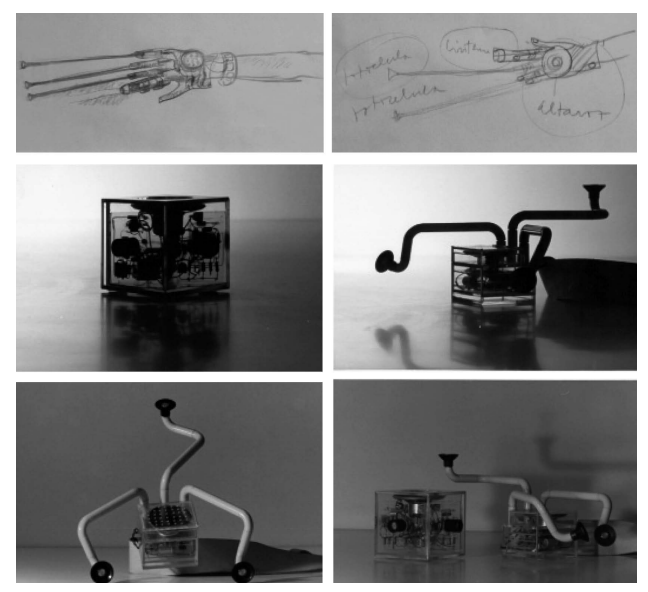

Fig. 2. Juan Navarro Baldeweg, Sounding Mirror (1971).

Fig. 3. Michio Ihara, Light Tower (1972) (izquierda). Juan Navarro Baldeweg, Square (1971), propuesta para el proyecto Times Square (derecha).

21. Véase la propuesta Reformed Traffic Circulation Pattern (1952) de Louise I. Kahn para Philadelphia (Pennsylvania).

22. Reconocida es la influencia que tuvo sobre esta primera pieza de Navarro Baldeweg Sculpture Cinétique (1920), de Naum Gabo, uno de los primeros artistas en manifestar su entusiasmo por la máquina, al que también se adscribieron Marcel Duchamp y Man Ray con Rotative plaques verre (1920) o Rotative demi sphère (1925) y László Moholy-Nagy con Light-Space Modulator (1921) que anticiparían el movimiento cinético.

23. En 1965, tan sólo en el Reino Unido existían seis centros de investigación que estaban explorando nuevas metodologías para su aplicación en el planeamiento urbano: Joint Unit for Planning Research, en Londres; Building Research Station, en Watford; Center for Urban and Regional Studies, en Birmingham; Institute for Community Studies, Land Use and Built Form Studies, en Cambridge y Geograhpy Department, en Londres.

24. NAVARRO BALDEWEG, J., SA1, Seminarios de Análisis y Generación Automática de Formas Arquitectónicas, op. cit., 1972.

25. Navarro Baldeweg se incorporó al CAVS como artista invitado por György Kepes para desarrollar su investigación posdoctoral, contando también con la supervisión de Nicholas Negroponte en aquellos temas de la investigación relacionados con el análisis de los sistemas urbanos desde el punto de vista cibernético.

26. NEGROPONTE, Nicholas, The Architectural Machine: Toward a more Human Environment, MIT Press, Cambridge, Massachusetts, 1973.
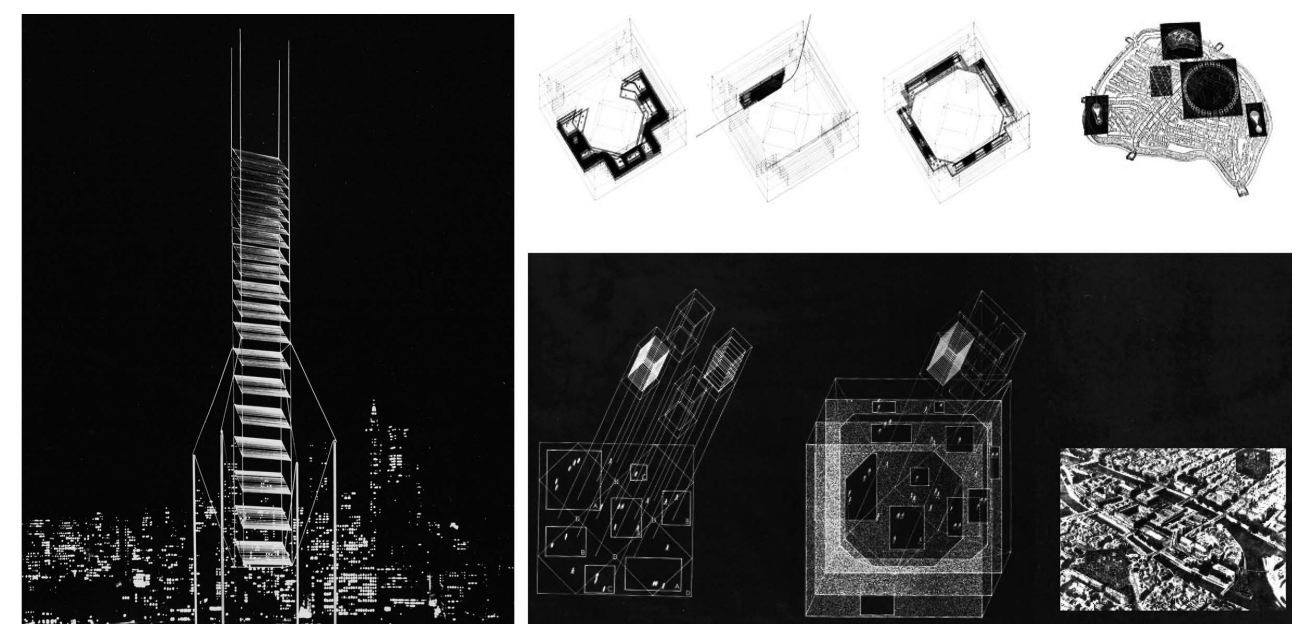

\section{PARTICIPACIÓN COLECTIVA EN LA OBRA ARTÍSTICA}

Otro de los fundamentos teóricos explorados por Kepes en el CAVS se refería a su creencia de que el individuo debía participar en la transformación del medio físico. Kepes consideraba que tanto la percepción y aprehensión de la realidad urbana como los procesos creativos asociados a su transformación debían implicar la participación de los ciudadanos en un proceso de organización formal, en el cual estuvieran asistidos por la tecnología, que podría poner a su alcance una serie de dispositivos multisensoriales que le permitirían interactuar con el medio ambiente.

Este interés en la participación colectiva para la planificación de entornos estaba presente también en la obra de Navarro Baldeweg, quien ya desde el comienzo de sus estudios de doctorado en Madrid en el año 1966 había entrado en contacto con nuevas metodologías de análisis urbano que buscaban relacionar las actividades que desarrollaban individuos y grupos sociales a lo largo del tiempo con la estructura física del entorno y su organización espacial, a partir de los planteamientos de Kevin Lynch, Gordon Cullen, Christopher Alexander o Louis I. Kahn, quien en su propuesta para Philadelphia ${ }^{21}$ analizaba los sistemas de comunicación urbanos asociándolos a energías dinámicas y bajo cuya influencia Navarro Baldeweg plantearía su primer sistema dinámico para resolver la congestión del tráfico, que ilustraría empleando una pieza cinética inspirada en las obras de Naum Gabo y László Moholy-Nagy ${ }^{22}$.

Más adelante, durante una estancia en Londres entrará en contacto con las obras de arte cinético y fenomenológico de David Medalla y Vassilakis Takis, en las que empleaban la variable tiempo como sustancia para coordinar energías latentes, acercándose con ello a los procesos analítico-científicos que se estaban empleando en centros como el Joint Unit for Planning Research ${ }^{23}$, un centro dependiente de la University College of London que funcionaba por entonces bajo la dirección de Peter Cowan, donde Navarro Baldeweg entraría en contacto con nuevas metodologías de análisis urbano que relacionaban la evolución temporal de las actividades sociales con el entorno urbano. En este contexto, su investigación doctoral tomaría una nueva dirección, que apuntaría hacia la necesidad de ampliar el concepto de sistema urbano a la noción de sistema artificial, entendido como herramienta de diseño que asegurase intervenciones sobre la ciudad consecuentes con las conductas sociales.

Estos presupuestos teóricos se materializarían en los sistemas artificiales que propondría durante su estancia en el Centro de Cálculo de la Universidad de Madrid entre 1969 y 1971, que se recogieron en la publicación $S A 1^{24}$ y le llevarían más adelante, influenciado por la teoría de sistemas de la información y la cibernética hacia la noción de sistema urbano entendido como sistema artificial, la cual materializaría durante sus primeros años de estancia en Boston bajo la influencia de Kepes y Nicholas Negroponte ${ }^{25}$. De hecho, Negroponte llegaría a publicar las propuestas de Navarro Baldeweg para la instalación Sounding Mirror (1971) en su libro The Architectural Machine: Toward a more Human Environment ${ }^{26}$, en el 

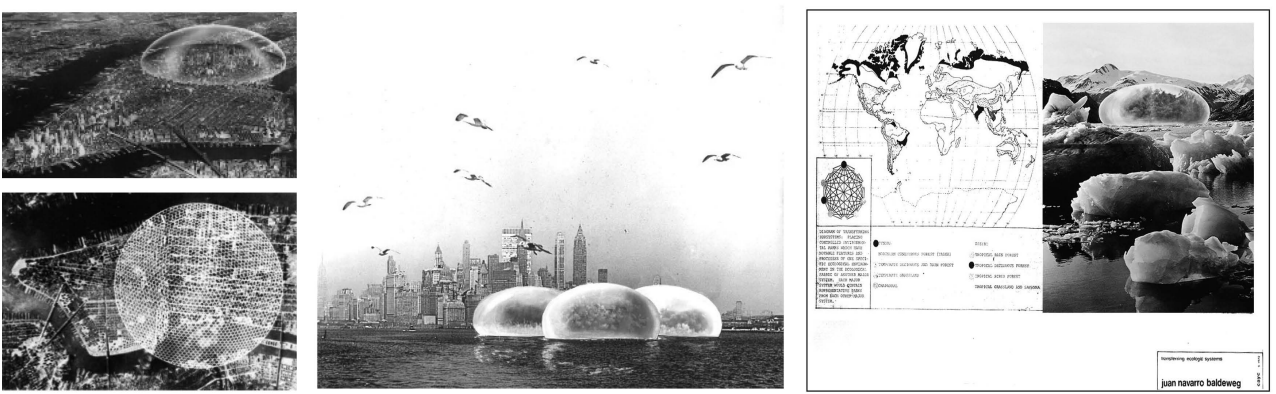

que trataba de redefinir la práctica de la arquitectura como una disciplina de carácter científico que permitiese la interacción del usuario en el proceso mediante el uso de la tecnología (Fig. 2). En el libro, más que presentar un modelo único, aportaba una colección de escenarios enfocados a dos objetivos fundamentales: en primer lugar, la definición del ordenador como una suerte de 'experto artificial', capaz de aportar a un usuario diferentes opciones en la definición del entorno urbano que derivasen en la solución más óptima adaptada a sus necesidades y en segundo lugar, la creación de una plataforma a modo de intermediario entre la comunidad y el arquitecto, buscando analizar las preferencias de los usuarios de cara a la planificación urbana.

Ambas líneas de trabajo también estaban presentes en Square -Plaza- (1971), un proyecto de Navarro Baldeweg para una dotación urbana para la plaza de Times Square realizado bajo la tutela de Kepes, en la que se proyectaban una serie de símbolos generados con luz láser que reflejaban los comportamientos de los usuarios (Fig. 3). Con ella pretendía abrir canales de información entre los individuos y la comunidad, registrando y transmitiendo información sobre las actividades que tenían lugar en el entorno urbano en tiempo real. La intención era crear unas 'puertas' o accesos a la información y unos mecanismos de decisión conjunta, que junto con las representaciones generadas pudieran llenar de significado ciertos intereses de orden social. Se trataba, en primer lugar, de incorporar al medio ambiente un sistema de símbolos o imágenes lo suficientemente variado como para representar en tiempo real procesos que tenían lugar en el ámbito de lo social y en segundo lugar, generar una estructura que permitiera la participación voluntaria de los miembros de la comunidad, el intercambio de información entre ellos y la creación y destrucción de símbolos de un lenguaje por determinar de forma participativa.

\section{ECOLOGÍA Y PAISAJE}

Con la idea de 'climax' ecológico Kepes se refería a un equilibrio entre el hombre y el entorno que planteaba como solución al deterioro medioambiental que se estaba produciendo por el crecimiento acelerado de las grandes ciudades. Para lograrlo llegaría a proponer a los artistas del CAVS el empleo de una serie de dispositivos capaces de permitir al individuo detectar posibles desequilibrios y actuar en consecuencia, estableciendo así una suerte de sistema de regulación ecológica. En el último volumen de la serie Vision and Value titulado Art of the Environment (1972)27, Kepes escribiría dos artículos en los que argumentaba la necesidad de reorientar la relación del individuo con su entorno ambiental, mediante la creación de sistemas artificiales basados en modelos perceptivos más complejos e inclusivos. En ellos demandaba la necesidad de proyectos artísticos dirigidos a reorientar la intervención en el medio con procesos y sistemas que tendieran hacia la desmaterialización del objeto, subordinando el trabajo con la materia física a la disposición y organización dinámica de energías y proponiendo, por tanto, la exploración sobre los procesos naturales en sus aspectos fenomenológicos. Para ilustrar estas consideraciones emplearía dos fotomontajes de Navarro Baldeweg denominados Proposal for the Increasing of Ecological Experience-Propuestas para el incremento de las experiencias ecológicas-(1972), en los cuales unas burbujas, entendidas como sistemas artificiales ecológicos, reproducían fragmentos del paisaje de ecosistemas que poseían cualidades medioambientales y especies vegetales características en diversas localizaciones (Fig. 4). El proyecto se inspiraba en los proyectos de Frei Otto, Richard Buckminster Fuller y Shoji Sadao para grandes cúpulas, pensadas para proteger extensas áreas urbanas creando
Fig. 4. Richard Buckminster Fuller y Shoji Sadao, Dome over Midtown Manhattan (1960) (izquierda). Juan Navarro Baldeweg, Ecosystems Floating in New York Harbor (1972) (centro y derecha).
27. KEPES, G., "The Artist's role in Environmental Self-Regulation", Arts of the Environment, George Braziller, New York, 1972. 
Fig. 5. Rockne Krebs, Suncase for Atlantis (1973) (izquierda) Juan Navarro Baldeweg, propuesta para Charles River (1971) (centro y derecha).

Fig. 6. Juan Navarro Baldeweg, Pneumatic Clouds over Boston (1971).
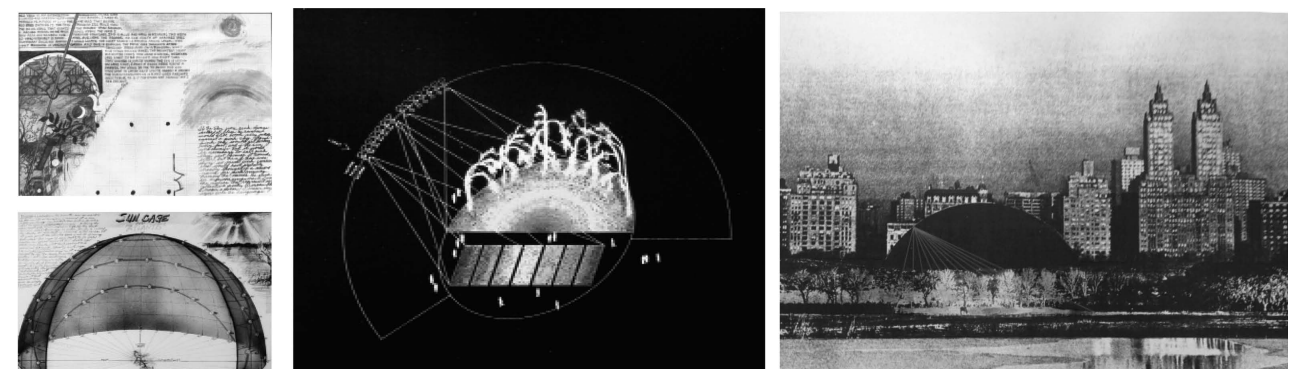

5
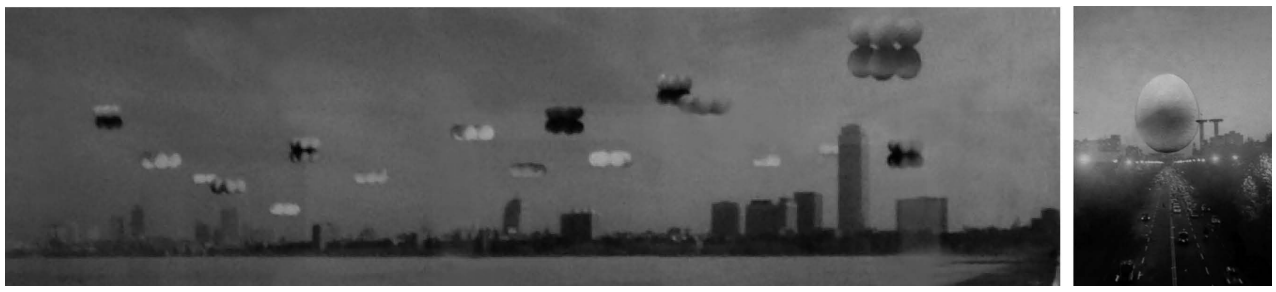

6

microclimas; en los proyectos de estructuras inflables que estaban desarrollando Timothy Johnson y Sean Wellesley Miller en el MIT y en las propuestas de intervención sobre el paisaje de Christo.

En la misma línea propondría otro sistema ecológico, un medio artificial creado encerrando un ecosistema en el interior de una cúpula semiesférica en el que se reproducía la morfogénesis de la vida vegetal. El proyecto planteaba la creación de un sistema adaptativo en el que se podía controlar la disposición y el tipo de las especies vegetales y que además, adquiría el papel de regulador de las condiciones del medio, garantizando su estabilidad y supervivencia. Con la propuesta se conseguía crear un ámbito en el que era posible activar algunos signos que mostraban las dependencias ecológicas entre la energía generada por la luz y el crecimiento de las especies vegetales que configuraban el espacio interior del jardín (Fig. 5). Se materializaban aquí dos conceptos que ya se habían empleado anteriormente para enunciar los principios de la teoría de la acción recogidos en "Acción y Diseño"28: la noción de sistema cibernético autorregulado de William R. Ashby $^{29}$-basada en el estudio de los sistemas biológicos, entendidos como sistemas homeostáticos y adaptativos- y la de ecosistema, para el que se basó en las investigaciones de Eugene Odum ${ }^{30}$.

En la misma línea de estos sistemas artificiales Kepes propondrá para el proyecto conjunto de regeneración de los márgenes del Charles River una serie de intervenciones que empleaban dispositivos de regulación medioambiental para reducir los altos niveles de polución y contaminación urbana. Algunos artistas proyectaron, para este mismo proyecto, sistemas de depuración de agua integrados en el paisaje que hacían visibles determinados procesos hidráulicos, los cuales además de responder a los requisitos funcionales, se establecían como símbolos reconocibles para la ciudad. Otros trabajaron con sistemas que hacían visibles los niveles de contaminación atmosférica o acústica provocados por la intensa actividad urbana, como Ted Kraynik, Mauricio Bueno, Bill Parker y el mismo Navarro Baldeweg, quien proyectaría Pneumatic Clouds over Boston -Nubes neumáticas sobre Boston- $(1971)^{31}$, unos dispositivos suspendidos en el aire a modo de apoyos informativos temporales que indicaban el grado de contaminación atmosférica y los niveles de tráfico en la ciudad, reflejando estos datos de forma visual según su agrupación y tonalidad (Fig. 6). Navarro Baldeweg. Una Caja de Resonancia, Editorial Pretextos, Colegio de Arquitectos de Cataluña, Barcelona, 2007.

35. Boston Harbor Project, Collaborative Projects, CAVS (1971-1974), op. cit. Center for Advanced Visual Studies Special Collection (CAVSSC), Massachusetts Institute of Technology, Program in Art, Culture \& Technology, Cambridge, Massachusetts.

36. NAVARRO BALDEWEG, J., "Proposal for the Charles River", Charles River Project (1971-73), op. cit., 1971.

\section{EXPLORACIÓN FENOMENOLÓGICA DEL MEDIO}

A comienzos de los años setenta se realizaron en el MIT numerosos proyectos orientados a solucionar los problemas medioambientales, destacando entre ellos los tutelados por Kepes en el CAVS y los coordinados por Negroponte en la School of Architecture and Planning, 

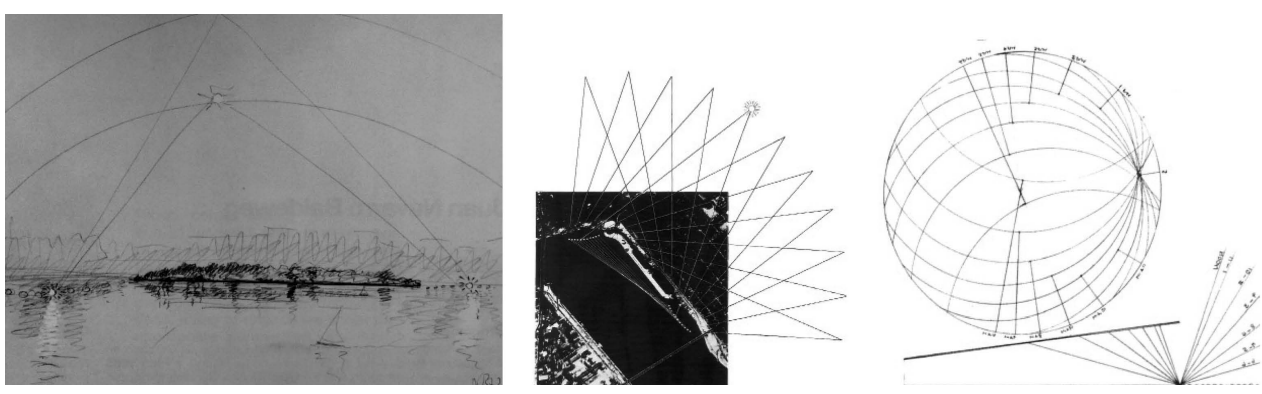

donde dirigía junto con Leon Groisser el Architecture Group Machine (Arch Mac) ${ }^{32}$. Si bien las investigaciones de Negroponte se dirigirían hacia una vertiente puramente tecnológica, buscando la aplicación de la cibernética en una serie de propuestas que terminarían desembocando en proyectos de inteligencia artificial, los proyectos del CAVS irían adquiriendo una orientación cada vez más dirigida a afianzar las relaciones entre el arte y la ciencia y a explorar las relaciones del individuo con la naturaleza desde el ámbito artístico. Tras los primeros años de estancia de Navarro Baldeweg en los Estados Unidos es posible apreciar una reorientación de su trabajo, que iría abandonando poco a poco las investigaciones sobre sistemas artificiales para centrarse en la exploración fenomenológica de la relación del individuo con el medio físico y en la manifestación y activación, que no reproducción, de dichas variables presentes en él, primero asistido por la tecnología y más adelante, a partir de pequeñas intervenciones que carecían de medios técnicos.

Así, comenzará a trabajar en una instalación con la que pretendía conectar al individuo a los ritmos de la naturaleza, haciéndole consciente del transcurrir del tiempo a partir de la idea de un reloj solar que permitiese visualizar las posiciones relativas de la tierra respecto al sol. La instalación formaba parte de las propuestas de intervención en el Charles River ${ }^{33}$ (Fig. 7) y estaba conformada por unas boyas ubicadas sobre la superficie del río, en las que se colocaban unos terminales que recibían la luz del sol canalizada a través de fibra óptica desde unos receptores situados en la orilla, de tal modo que desde la salida del sol hasta el ocaso se iban encendiendo e iban marcando las horas. La instalación mostraba el paso del sol hecho visible por el encendido secuencial de cada una de las terminaciones, quedando el recorrido del sol dibujado sobre la superficie del agua y definiendo lo que Navarro Baldeweg asociaría con el "derrape de la tierra en el espacio soleado". 34

Esta idea de materializar los ciclos temporales de la naturaleza empleando intervenciones sobre un emplazamiento específico, entendidas como articulaciones conceptuales o 'puentes' entre el individuo y el medio físico, también se encontraba presente en las propuestas de otros artistas del CAVS como Keiko Prince, quien trataría de reflejar la percepción del tiempo a partir de fenómenos naturales o Bill Parker y Alejandro Otero, quienes trataban de hacer perceptibles los cambios de luz durante el recorrido del sol. También orientadas a la recuperación de la zona del muelle histórico de Boston, conocida como Long Wharf ${ }^{35}$, plantearían instalaciones para la activación de variables naturales en determinados lugares que se entendían como espacios de transición. Entre ellas destacan las proyectadas por Keiko Prince, Maryanne Amacher y Luis Frangella, quienes plantearon una serie de ambientes sonoros, proyectados con la idea de recrear un sentido de lo oculto presente en un lugar, combinando percepción auditiva y visual. Kepes también planteó un proyecto para la reintegración de las islas de Boston Harbor en el que trabajaron Lowry Burgess y Navarro Baldeweg, quien propondría una instalación en la que materializaría el movimiento del sol a lo largo del día en las distintas estaciones del año, creando una suerte de cartografía solar. Para ello, emplearía los receptores que utilizó en el proyecto del Charles River, aunque en esta ocasión, situados en dos islas desde donde se transportaba la luz del sol a unas boyas dispuestas en el agua, que hacían visible el recorrido del sol ${ }^{36}$ (Fig. 8).

En la misma línea de estas propuestas, aunque ya liberado de la tecnología, Navarro Baldeweg comenzaría a trabajar en una serie de piezas e instalaciones sobre habitaciones vacías que lograban registrar el recorrido de la luz durante su movimiento a lo largo del día pero
Fig. 7. Juan Navarro Baldeweg, Skiding Sky (1971), propuesta para Charles River (izquierda y centro). Keiko Prince, The Shelter: Sun Water-Dial (1971) (derecha).
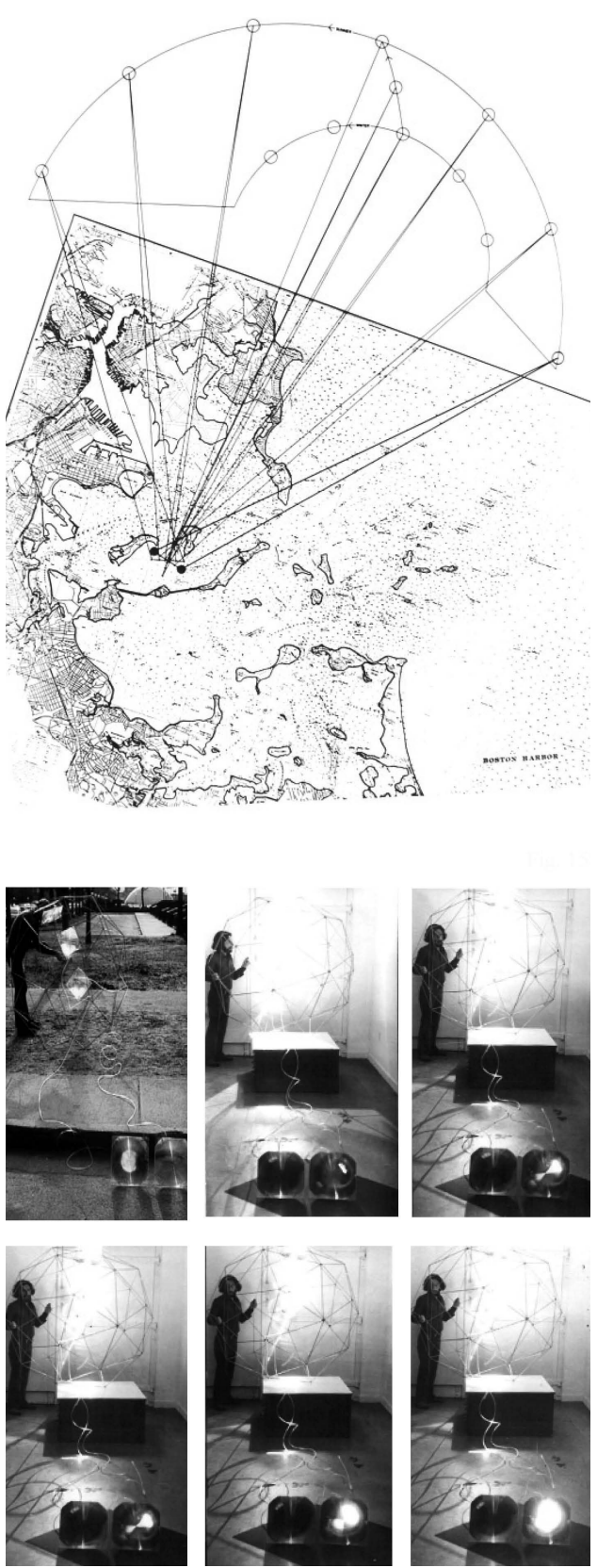

Fig. 8. Juan Navarro Baldeweg, Skiding Sky (1972), planos y fotografías del proyecto para Boston Harbor. 
Fig. 9. Juan Navarro Baldeweg. Pieza de sombra en forma de libro -Source and Sink-(1973).

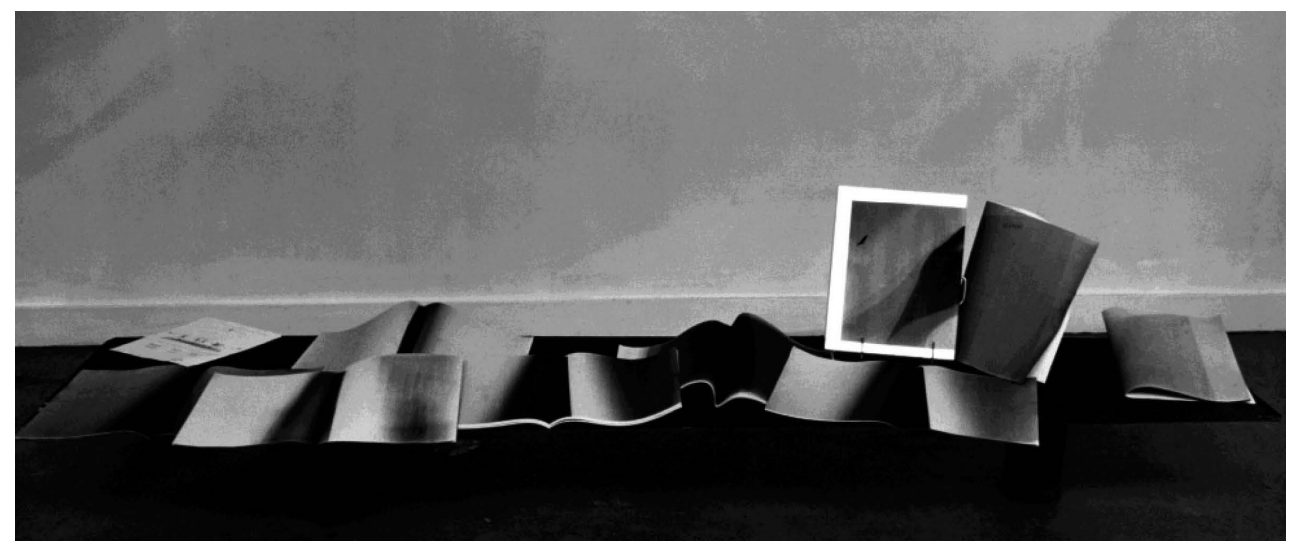

sin incorporar medios tecnológicos sofisticados. Así, en Fuente y fuga-Source and Sink$(1973)^{37}$, apoyándose únicamente en acciones de interposición de sustancias o entidades, como unas páginas de papel sensible, conseguía materializar el flujo continuo de luz natural de una habitación inscribiéndola en el ciclo solar, al registrar sobre el papel el recorrido de la luz (Fig. 9). Durante los dos últimos años de su estancia en el CAVS realizaría varias piezas e interiores en los que quedaba patente este deseo de hacer tangible la vinculación entre el individuo y su entorno y que más adelante trasladaría al ámbito de lo arquitectónico en el primer proyecto que realizó a su regreso a Madrid: la Casa para una Intersección (1976) ${ }^{38}$. En ella pondría en práctica todas las experiencias con la activación de signos que había desarrollado en su etapa americana, forjando un modo de proyectar que se apoyaría en el establecimiento de una continuidad entre el individuo y la naturaleza, a través de su conexión con los ciclos naturales.

\section{DE LA FIGURACIÓN A LA ABSTRACCIÓN}

Para Kepes existían dos niveles de aproximación a la transformación del medio ambiente urbano desde el ámbito creativo. El primero se refería al interés privado y ofrecía nuevas estrategias para intervenir desde el punto de vista formal sobre la ciudad. El otro, en cambio, trabajaba a un nivel más profundo y menos legible proponiendo nuevos modos de experimentar la ciudad, a partir de aproximaciones más conceptuales que pudieran venir seguidas de experiencias sensibles ${ }^{39}$. A este segundo nivel asociaría la propuesta The Anywhere City $(1974)^{40}$ de Maryanne Amacher, Luis Frangella y Navarro Baldeweg, quienes basándose en la idea de que la relación del hombre con el medio físico se veía constantemente alterada por la interpretación que éste hiciera de ella, consideraban que el reconocimiento de un ámbito requería la construcción por parte del individuo de sus propios mapas, en los cuales reflejase las conexiones entre sus ideas o proyectos y la realidad. Según esta idea, la propuesta planteaba la realización de sutiles intervenciones a modo de puertas metafóricas, a través de las cuales el mundo interior propio de cada individuo pudiera proyectarse hacia la realidad física, configurándose con ello una suerte de mapa que registrase dichas puertas o pasajes sobre la ciudad existente. Éstas permitirían el acceso a otras realidades inmateriales a través del cuerpo y la mente del individuo. La exploración del medio se realizaba a partir de actuaciones mínimas en habitaciones vacías, situadas en lugares ubicados en determinadas áreas urbanas, en las que fuese posible crear imágenes sensibles que evocasen los mundos interiores presentes en la imaginación de cada individuo. Con ello materializaban dos ámbitos íntimamente relacionados entre sí, una habitación real, de la que se extraían una serie de variables esenciales y otra perteneciente al mundo de la imaginación, donde éstas quedaban materializadas, gracias a la cual era posible apreciar valores sensitivos acústicos o perceptivos- que de otro modo pasaban desapercibidos, ampliando el umbral de percepción visual o sonora de un ámbito.

En los artículos "Habitaciones" ${ }^{41}$ "Simbolismo de centro y pasaje en el entorno"42 Navarro Baldeweg profundizaría sobre esta correspondencia entre lo real y lo imaginario, pero donde realmente se aprecia esta idea es en Interior 1 (1973), una instalación en la que construía un ámbito imaginario en la entidad física de una habitación vacía a partir de 

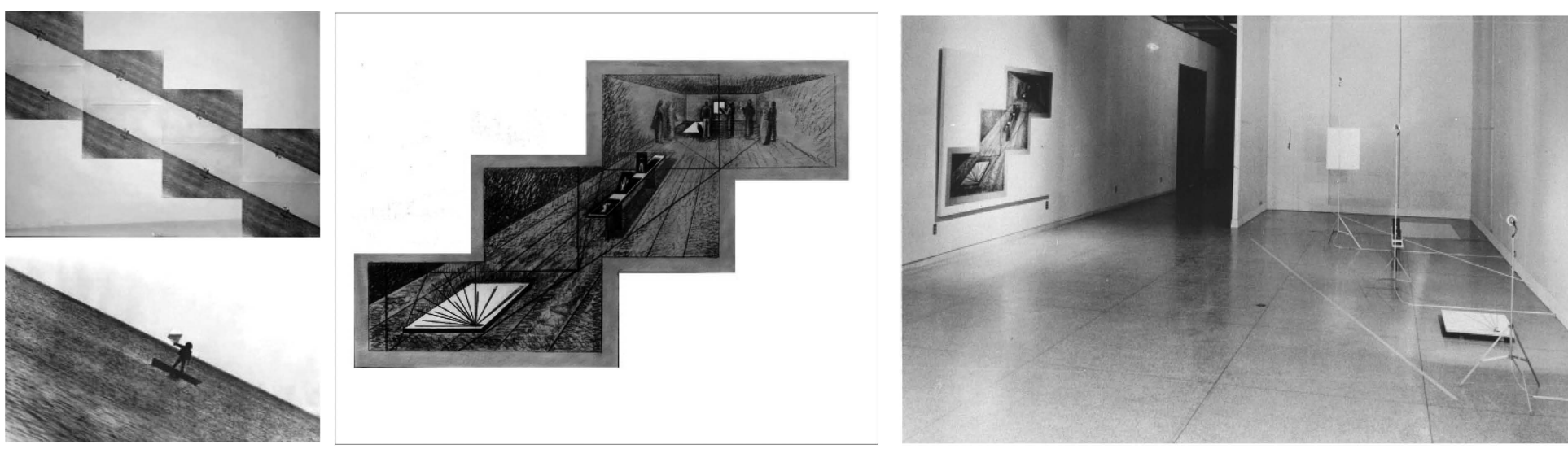

10

mínimas intervenciones, que dotaban a este espacio de complejidad semántica o simbólica (Fig. 10) ${ }^{43}$. La instalación podía entenderse como un pasaje, un ámbito de transición representado a partir de una secuencia entre tres estados identificados con una escena, su analogía y la abstracción. En el primero se dispusieron unas intervenciones en las que se permitía a los espectadores explorar su relación con la luz y el espacio; en el segundo se establecía un paralelismo con la anterior, a partir de tres fotografías que ilustraban tres modos de interposición de un sujeto en el flujo de la luz y por último, en el tercero, se recogía la sombra de unas varillas metálicas interpuestas en el flujo de la luz. Esta condición de la obra como tránsito entre la realidad física y la abstracción geométrica quedaba reforzada con la colocación en la habitación del dibujo empleado por Navarro Baldeweg en la creación de este primer interior, una perspectiva que representaba el espacio activado. Desde aquel primer interior realizaría otros cuatro más, entendidos como ámbitos en los que explorar las correspondencias entre un espacio mental, en el que se desarrollaban los procesos de pensamiento y un espacio real: el mundo físico.

La existencia de estos dos mundos, la realidad física y el mundo de la imaginación se aprecia también en la obra Juliet (1937) de Kepes, una fotografía de su mujer mirando ensimismada a través de una ventana. La luz filtrada sobre su rostro y su actitud reflexiva a la que se refieren unas imágenes superpuestas sobre la fotografía, que parecen materializar el hilo que siguen sus pensamientos, reflejan esa habitación mental a la que se ha venido refiriendo Navarro Baldeweg en sus escritos y a la que se accede a través de la contemplación del medio físico. Aquí, la ventana se entiende como elemento que conecta dos ámbitos complementarios: las coordenadas de la realidad física y la mente. Esto se aprecia también en Edge of the Sun (1975), una fotografía de Navarro Baldeweg del rostro de una mujer con una mano sobre la frente, con la que parece protegerse de la luz del sol que baña parcialmente su cara (Fig. 11). Su frontalidad implica una simetría entre la mitad del rostro en el que se aprecian nítidamente sus rasgos físicos y la otra mitad, medio en penumbra, donde se percibe un rostro desmaterializado, en el que se distinguen unas manchas de luz que representan el rastro del sol en el discurrir del día y que registran el efecto de una acción en el transcurso del tiempo. Aparece aquí de nuevo este transitar entre la realidad, representada por la parte del rostro reconocible y la abstracción, identificada como la parte del rostro desmaterializada por efecto de la sombra.

\section{VARIABLES ESENCIALES, COORDENADAS DEL HOMBRE}

Durante su última etapa en el CAVS Navarro Baldeweg se centraría en una serie de obras pensadas para la activación del medio ambiente, realizadas a partir de unas piezas sencillas que no incorporaban sofisticadas tecnologías. Comenzó trabajando con unas piezas de luz creadas colocando unas telas de terciopelo suspendidas en el aire, en las que situaba una gota de aceite, de forma que ésta se encendía al recibir la luz natural transformándose en una señal o un signo. A partir de éstas realizaría varios interiores en los que propondría mínimas intervenciones en una habitación vacía, que dotaban al lugar de cierta complejidad semántica. Así, tras el mencionado Interior I (1973), en el Interior II (1974) un conjunto de piezas de sombra, gravedad, magnetismo y tiempo se dispondrían para configurar un ámbito en el que cada sistema evidenciaba la existencia de un flujo
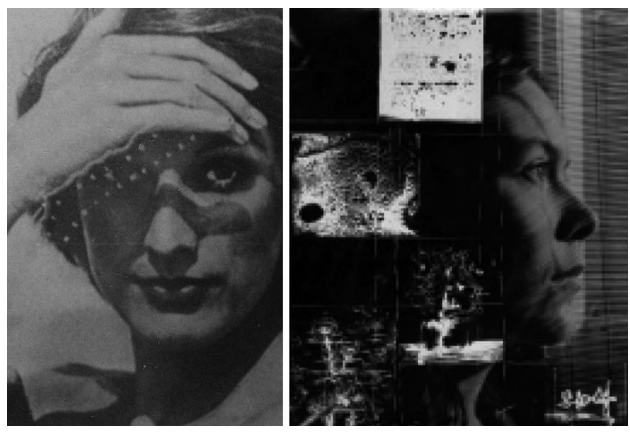

11

Fig. 10. Mauricio Bueno, Horizontes (1973) (izq). Juan Navarro Baldeweg, instalación Interior I (1973) (centro y dcha). Fig. 11. Juan Navarro Baldeweg, Edge of the Sun -Orilla de Sol- (1975) (izq). György Kepes, Juliet (1937) (dcha).

43. "La propuesta es una indagación en la aplicación de algunos procesos de la imaginación en el mundo físico; un experimento sobre la correspondencia entre un espacio interior, en el que tienen lugar los procesos de pensamiento, ensoñamiento e ideación y el espacio real". NAVARRO BALDEWEG, J., "Interior I", Individual Works by Fellows at the Center for Advanced Visual Studies 1973-74, op. cit. 
Fig. 12. Juan Navarro Baldeweg, Interior II (1974) (izquierda). Juan Navarro Baldeweg, Interior III (1975) (derecha).
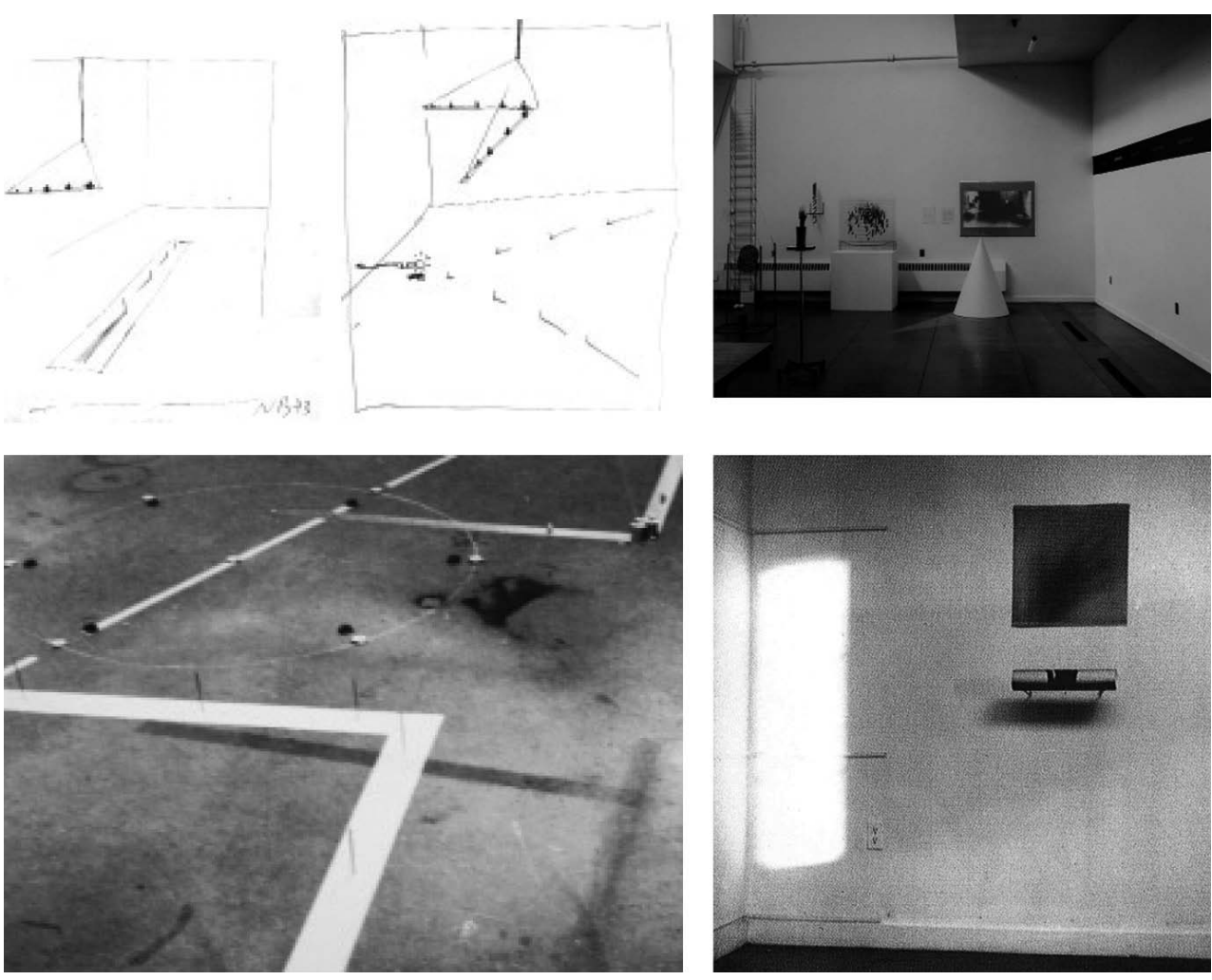

energético y su disposición conjunta permitía establecer correspondencias y asociaciones entre todos ellos. A partir de aquí realizaría otras piezas de gravedad, preludio de las que ha continuado realizando hasta el día de hoy, que plantean la activación del campo gravitatorio presente de manera permanente en el medio físico, a partir de una serie de signos mínimos sensuales que hacen visibles energías naturales. Kepes pensaba que estas piezas estaban bajo el propósito de activar signos de un sistema físico básico, lo consideraba un trabajo sustanciado en ciertas coordenadas esenciales que proporcionaban experiencias directas de la naturaleza y del propio cuerpo, facilitando el paso a un mundo interno abierto por los sentidos a través de ellas.

Las instalaciones relacionadas con la luz y la gravedad pusieron de manifiesto su capacidad para para activar signos en un medio a partir de mínimas intervenciones, aunque en este proceso de activación el sujeto no participara de un modo activo, ya que al provocar sensaciones que llegaban a los sentidos producían generalmente una recepción de carácter pasivo. Esta reflexión le llevaría a establecer una distinción entre 'sensación', entendida como aquello que llega a los sentidos a través de la percepción e identificada como algo pasivo y la 'expresión', definida como algo que se realiza a través del gesto y en consecuencia considerada como activa ${ }^{44}$. Es entonces cuando adquiere la conciencia de que la 'sensación' y la 'expresión' son dos fronteras del mismo cuerpo y que se identifican con la percepción y la gestualidad y aparecen aquí reflejados, por primera vez, las nociones de las cuatro variables esenciales del medio que terminaría denominando más tarde como luz, gravedad horizonte y mano.

La necesidad de plasmar la huella del cuerpo en el ámbito de la habitación le llevaría a proponer Interior III (1975), una instalación en la que una habitación se configuraba para la creación de after images, poniendo de manifiesto como su activación quedaba supeditada a la energía del cuerpo del individuo al situar la fuente de generación de las imágenes en la misma base del mecanismo perceptual del ojo (Fig. 12). En Interior IV (1975), influenciado por las instalaciones de Sol Lewit, comenzaría a superponer directamente sobre las paredes de la habitación trazos manuales, los cuales avanzarían los trazos de colores empleados en Interior V (1976), donde además es posible encontrar un compendio de todos los temas o motivos recurrentes en los que había trabajado durante aquellos años. Allí se agruparon fotografías como Fuente y fuga (1973), La T y la sombra (1973), La columna y el peso (1973)

44. "La experiencia de la luz es pasiva. La experiencia de la gravitación es pasiva. La experiencia del agua, del viento, de los fluídos es pasiva. (...) Pero había otro movimiento de dentro hacia afuera, que en alguna ocasión yo he llamado ciente, la huella de tu cuerpo, de tu mano. (...) Yo . como una necesidad, como una coordenada vital tan esencial como las otras". ZUAZNABAR, Guillermo, "Conversaciones con Juan Navarro Baldeweg", Conversaciones con estudiantes, Gustavo Gili, Barcelona, 2011. 

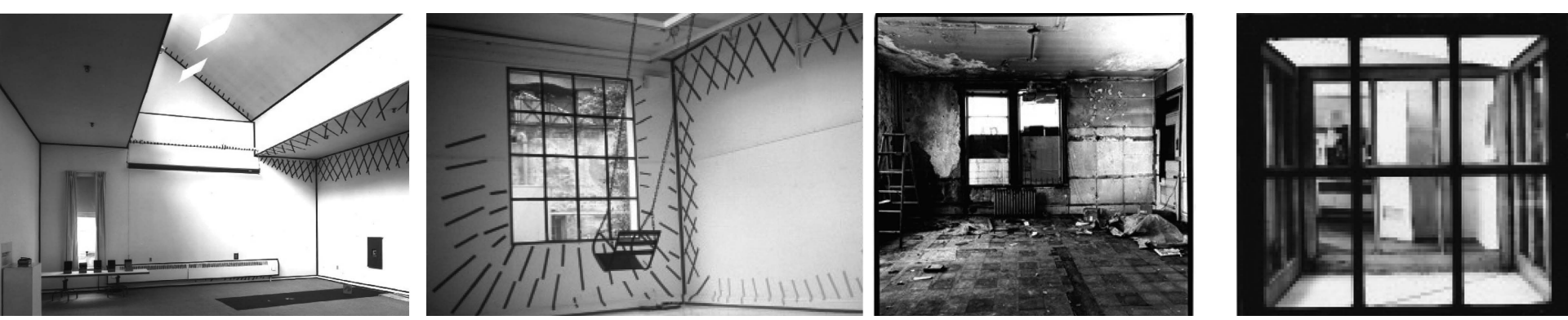

u Orilla de sol (1975); los dibujos del proyecto para la Casa para una Intersección (1976) y obras como Arado (1975), Cinco unidades de luz, cinco habitaciones (1974) o Acción de los sombreros (1975) junto con otras inéditas que venían a sintetizar el trabajo de la etapa americana (Fig. 13). En la exposición se agruparían por primera vez sus variables esenciales en cuatro categorías, la luz y la gravedad, vinculadas a los mecanismos perceptivos que el individuo recibía de un modo pasivo y el horizonte y la mano, las cuales se relacionaban con los mecanismos creativos que implicaban a un sujeto en acción. Quizá debido a que ambas, la exploración visual y el hacer, requerían de un cuerpo que interviniese activamente en el medio físico, Kepes llegaría a denominar a estas variables esenciales como las 'coordenadas del hombre' que proporcionaban experiencias directas de la naturaleza que rodea a cada individuo y de su propio cuerpo.

\section{CONCLUSIONES}

Con la serie de interiores Navarro Baldeweg sentaría las bases de lo que con los años llegaría a definir como una 'habitación excitada', un ámbito cuyo objetivo único sería la celebración de las coordenadas esenciales. En ellos ponía de manifiesto el carácter sintético de las instalaciones, en el sentido de que agrupaban en un único recinto, piezas de muy variada naturaleza que había ido asociando a varias categorías según las dimensiones del medio físico que éstas activasen. Allí lograría situar en convivencia piezas de distintas categorías, presentadas de tal forma que las significaciones asociadas a cada una de ellas se multiplicaban debido al diálogo que establecían entre ellas. Con ello conseguiría explicar también el carácter transitivo de sus obras, que se activaban a partir de la percepción de un sujeto y a su vez dialogaban con el contexto en el que se inscribían, de modo que ambos pasaban a formar parte de la obra.

Una mirada sobre la obra realizada por Navarro Baldeweg a lo largo de toda su trayectoria profesional pone de manifiesto su adscripción a los presupuestos teóricos que allí se presentaron, explorados durante aquellos años de estancia en el CAVS bajo la tutela de György Kepes. En las retrospectivas que han mostrado su trabajo a lo largo de estos últimos años, se puede apreciar su capacidad para representar la realidad a partir de distintas manifestaciones artísticas y su interés por mostrar todas ellas compartiendo un mismo espacio, algo que está presente ya en esta primera etapa en la que trabajó con distintos soportes y medios, tratando de atender a la realidad con la misma variedad con la que se le presentaba. Por otra parte, una revisión de su obra plástica y arquitectónica pone de manifiesto su constante deseo de ahondar aún hoy en el conocimiento del medio físico, a partir de la relación que establece el individuo con el espacio que le rodea, proponiendo para ello diversos modos de interacción del cuerpo con el entorno, a partir de distintas intervenciones sobre el medio como instalaciones, pinturas y obras de arquitectura.

En toda su obra se hace patente también algo ya presente en los proyectos de aquellos años: una atención explícita hacia la exploración de las relaciones de los individuos con el mundo que les rodea y hacia los modos de aprehensión de la realidad física. Durante su estancia en el CAVS inició un camino, en el que despojándose de todo medio instrumental, buscaba desarrollar una actividad creativa de estética austera fundamentada en la pura contemplación, que le permitiera desvelar variables ocultas de un ámbito espacial. Con ello ha logrado definir a lo largo de los años, una estructura conceptual que le ha permitido comprender el espacio urbano y arquitectónico, a partir de un sistema de coordenadas basado en cuatro variables esenciales: luz, gravedad, horizonte y mano. Dichas variables, denominadas
Fig. 13. Juan Navarro Baldeweg, Interior IV (1975) (izquierda). Juan Navarro Baldeweg, Interior V (1975) (centro). Maryanne Amacher, City Links (1967-1978) y Luis Frangella, The Window (1973) (derecha). 
como 'coordenadas del hombre' por el propio Kepes, plantean la existencia de un espacio mental asociado a la realidad física y suspendido en el tiempo, al alcance de aquellos individuos dispuestos a implicarse en su aprehensión. Esta idea de que la percepción de este espacio mental está íntimamente vinculado al propio ser del individuo, que ha materializado tantas veces a lo largo de su trayectoria en sus pinturas, instalaciones y proyectos de arquitectura tuvo su origen en estas primeras investigaciones, donde ya incitaba al espectador a participar del ámbito propuesto en sus interiores, a partir de sutiles intervenciones que incidían directamente sobre los sentidos e incitaban a la participación del individuo en la definición de este espacio complementario.

Covadonga Lorenzo Cueva. Doctora Arquitecta por la Universidad de Navarra, Arquitecta por la Universidad Politécnica de Madrid y Máster en Diseño Arquitectónico por la Universidad de Navarra. Durante seis años formó parte del equipo editorial de la revista Arquitectura Viva \& AV Monographs y actualmente dedica su trabajo a la docencia y la investigación en la Universidad CEU San Pablo, donde ha ejercido como Coordinadora de Relaciones Internacionales y Subdirectora de Organización y Recursos Académicos. Actualmente es Directora del Laboratorio de Fabricación Digital; profesora del Departamento de Arquitectura y Diseño y Jefa de Redacción de la revista de arquitectura Constelaciones. Sus publicaciones más relevantes están relacionadas con el tema de su tesis doctoral titulada Juan Navarro Baldeweg en el Center for Advanced Visual Studies del MIT (1971-1975), que analiza la investigación desarrollada por Navarro Baldeweg durante la primera etapa de su trayectoria profesional, orientada a la intervención sobre el medio ambiente a partir de propuestas teóricas, cuya recuperación resulta relevante hoy en día, no sólo por la transcendencia que tuvieron en sus trabajos posteriores, sino también por la actualidad de los temas tratados. 